\title{
Siliceno, una nueva mirada al silicio en dos dimensiones*
}

\author{
Pamela Rubio-Pereda**, Noboru Takeuchi***
}

RESUMEN: El siliceno, un material bidimensional de estructura hexagonal similar al grafeno, ha estado recientemente en la mira de la comunidad científica de las nanociencias debido a que podría extender por mucho las posibles aplicaciones del silicio, elemento usado por excelencia desde hace más de medio siglo en la industria electrónica. Esta novedosa estructura bidimensional promete ofrecernos novedosas aplicaciones en la siguiente generación de componentes electrónicos, en la que predominarán dispositivos flexibles que revolucionarán la tecnología actual. Al igual que en el caso del grafeno, la cantidad de investigaciones teóricas acerca de esta prometedora estructura, se ha visto incrementada hasta abordar incluso el tema de la funcionalización de su superficie mediante la incorporación de diferentes elementos, para incluso sugerir aplicaciones en el ámbito de los sensores. Actualmente, diversos grupos de científicos experimentalistas se encuentran sintetizando esta estructura mediante el empleo de diferentes técnicas. Los resultados de ambos experimentos teóricos y experimentales han propiciado una profusa cantidad de publicaciones, que han abierto nuevas líneas de investigación en torno a esta novedosa nanoestructura. PALABRAS CLAVE: Siliceno, estructura hexagonal, nanoestructura.

ABSTRACT: Silicene, a hexagonal two-dimensional material similar to graphene, has recently become the topic of active research in the field of nanoscience. Silicene could extend the possible applications of silicon, which in the last half century has been the most important material used by the electronics industry. This novel two-dimensional material promises to offer new applications in the next generation of electronic components, in which flexible devices will revolutionize the current technology. As in the case of graphene, the amount of theoretical investigations on this promising structure has increased very quickly. It is now a topic of interest, the study of the functionalization of the surface by adding different elements that could lead to applications in the field of sensors. Currently, several experimental groups are trying to synthesize this structure by employing various techniques. The results of both theoretical calculations and experimental methods have led to a profusion of publications, which have opened new lines of research for this novel nanostructure. KEYwORDS: Silicene, hexagonal structure, nanostructure.

Desde finales del siglo pasado, la investigación en nanociencias y nanotecnologías ha estado grandemente influenciada por estructuras hexagonales a base de carbono con múltiples dimensionalidades. Como ejemplo de estas estructuras, conocemos los fulerenos, los nanotubos de carbono en todas sus variedades $\mathrm{y}$, recientemente descubierto, al grafeno como una de las tantas capas individuales que conforman al grafito.

\footnotetext{
* Se agradece a DGAPA proyecto IN103512-3 y Conacyt Proyecto 164485 el apoyo económico. Los cálculos se han realizado en el Centro de Supercómputo de la DGCTIC-UNAM.

** Centro de Investigación Científica y de Educación Superior de Ensenada, Apartado Postal 2681, Ensenada, Baja California 22800, México.<rubio.pereda@gmail.com>.

***Centro de Nanociencias y Nanotecnología, Universidad Nacional Autónoma de México, Apartado Postal 2681, Ensenada, Baja California 22800, México.<takeuchi@cnyn.unam.mx>.
} 
El grafeno descubierto en 2004 (Novoselov et al., 2005), por Geim Novoselov y colaboradores en la universidad de Manchester, exhibe ciertas propiedades electrónicas y mecánicas que lo han colocado como una de las estructuras más conocidas en el mundo científico (Martínez Guerra et al., 2009). Debido al gran potencial que presenta el grafeno, se ha generado una rica veta de investigaciones tanto teóricas como experimentales, que lo posicionan como la nueva superestrella de los nanomateriales.

Los recientes éxitos asociados al grafeno han conducido, en primera instancia, a la investigación teórica de estructuras con base hexagonal formadas por otros elementos. Para esto, diversos grupos de investigación se han focalizado en estudiar elementos que presenten una configuración electrónica análoga a la del elemento carbono. Estos elementos que han sido abordados y que conforman parte del grupo IV de la tabla periódica, bien denominado el grupo de la familia del carbono, son el silicio y el germanio.

Sin embargo, las primeras investigaciones teóricas realizadas en tales sistemas bidimensionales fueron llevadas a cabo incluso 10 años antes del descubrimiento del grafeno, motivadas por las primeras síntesis que se realizaron en la elaboración de diversos compuestos con silicio que presentaban propiedades similares a las olefinas, compuestos con al menos un doble enlace carbono-carbono (West et al., 1981).

En 1994, Takeda y Shiraishi estudiaron las características estructurales de sistemas bidimensionales hexagonales a base de silicio (Takeda y Shiraishi, 1994), mediante el uso de cálculos de energía total de primeros principios. A partir de tales cálculos, se encontró que el silicio efectivamente puede presentar una estructura bidimensional, aunque a diferencia del grafeno, esta estructura presentaría cierto grado de deformación atribuida principalmente a una gran contribución repulsiva proveniente del potencial de la red.

Cálculos de primeros principios, efectuados por Cahangirov y colaboradores en el 2009 con la teoría del funcional de la densidad (Cahangirov et al., 2009), mostraron que el siliceno en realidad puede presentar tres posibles configuraciones para una estructura bidimensional, la cual puede mostrarse como una red plana, no plana con bajo grado de deformación (LB por sus siglas en inglés, low buckled) y no plana con alto grado de deformación (HB por sus siglas en inglés, high buckled), con desplazamientos verticales promedios de $0 \AA$, $0.44 \AA$ y $2.13 \AA$, respectivamente, entre átomos de silicio contiguos.

Como se observa en la figura 1, la configuración de un siliceno con una red plana, resulta energéticamente menos favorable que la de un siliceno con una red con cierto grado de deformación. La estabilidad real entre las estructuras de siliceno HB y LB es evaluada al estudiar la dispersión de fonones de las redes respectivas. Como resultado de esto, el siliceno LB resulta ser una estructura estable, mientras que el siliceno HB no es una estructura estable, debido a que los modos de dispersión de fonones de esta estructura se muestran en frecuencias imaginarias.

La estructura del siliceno LB (figura 2) resulta en una red bidimensional de átomos de silicio dispuestos en una celda hexagonal con un parámetro de red $a$ equivalente a aproximadamente $3.87 \AA$ A. Por cada celda unitaria existe un punto de red al cual se asocian dos átomos de silicio, en donde éstos se encuentran desplazados verticalmente uno del otro una distancia aproximada de $0.44 \AA$.

A diferencia del grafeno que muestra una alta preferencia por una configuración tipo trigonal plana con un alto grado de enlazamiento tipo $\sigma$ y $\pi$ entre orbitales atómicos, el siliceno, con una red deformada, presenta un menor traslape entre orbitales atómicos, 
FIGURA 1. Variación en la energía total para estructuras hexagonales bidimensionales de $C$ y Si con respecto a la constante de red $a$ en Å de la celda unitaria. Cálculos obtenidos a través de primeros principios con la teoría del funcional de la densidad.

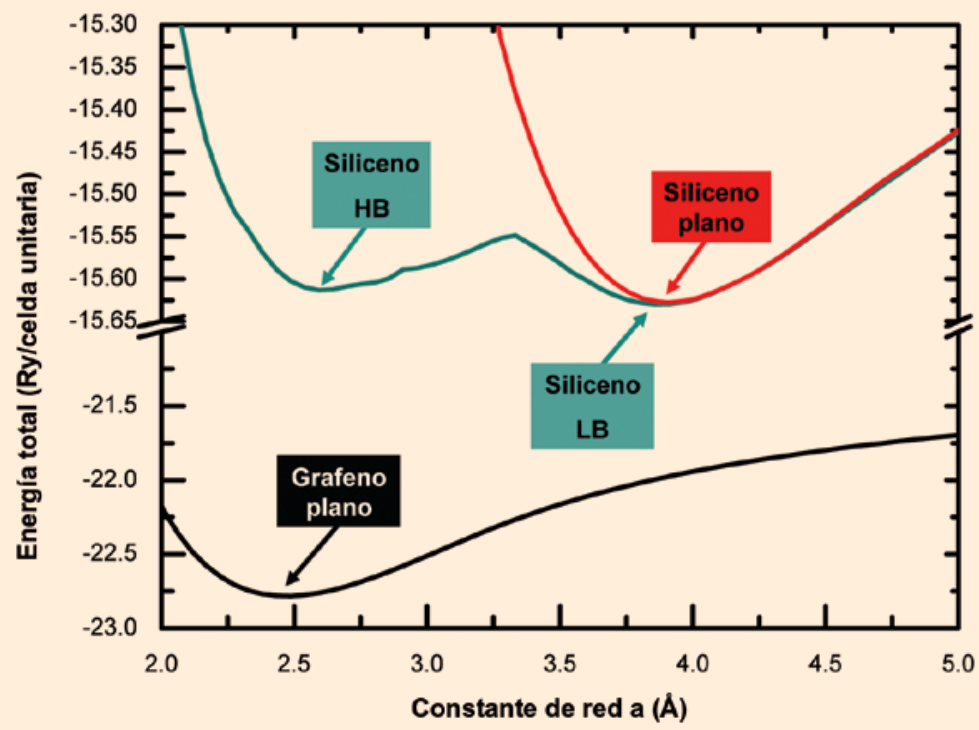

FIGURA 2. Vista transversal y en el plano del grafeno y siliceno. En la estructura de grafeno los átomos de carbono están ubicados en un solo plano; sin embargo, en la estructura deformada de siliceno, los átomos se alternan en planos paralelos.

\section{Grafeno}

\section{Siliceno}
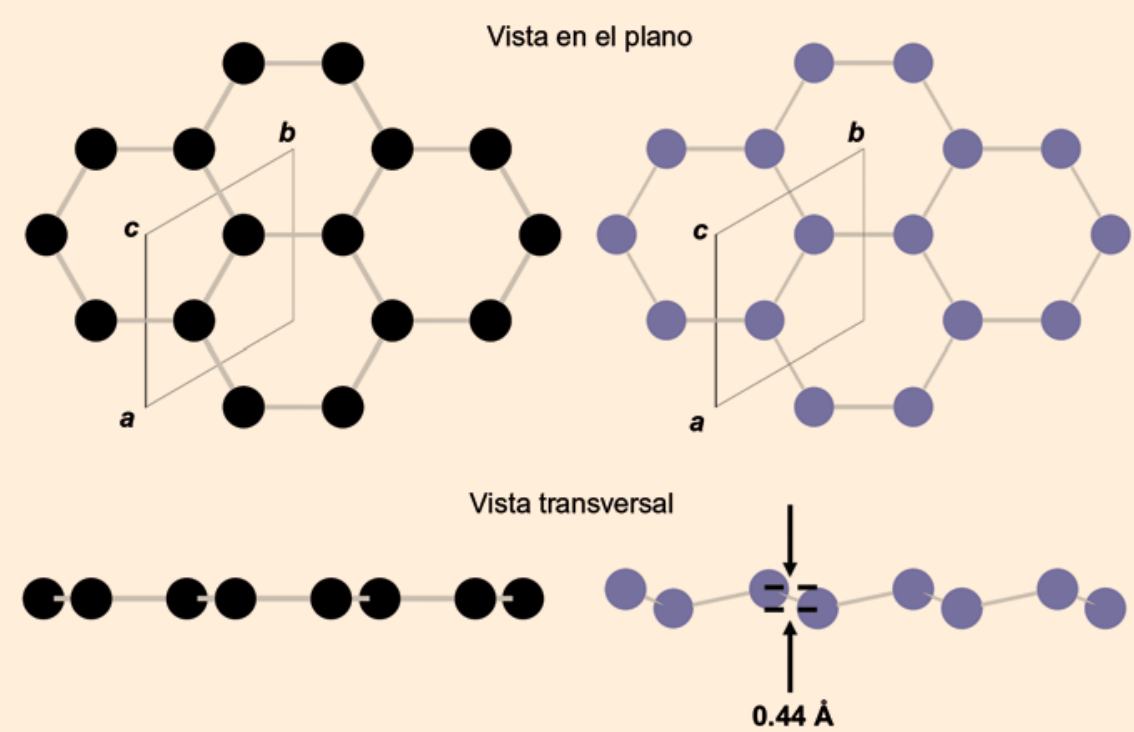
FIGURA 3. Mapas de densidad electrónica para los enlazamientos $\sigma$ y $\pi$ en estructuras con geometría plana y deformada, características del grafeno y siliceno, respectivamente. El incremento en la densidad de carga es graficado con colores amarillo para una densidad tenue y con rojo para una densidad prominente. Figura adaptada de Sahin et al., 2009.

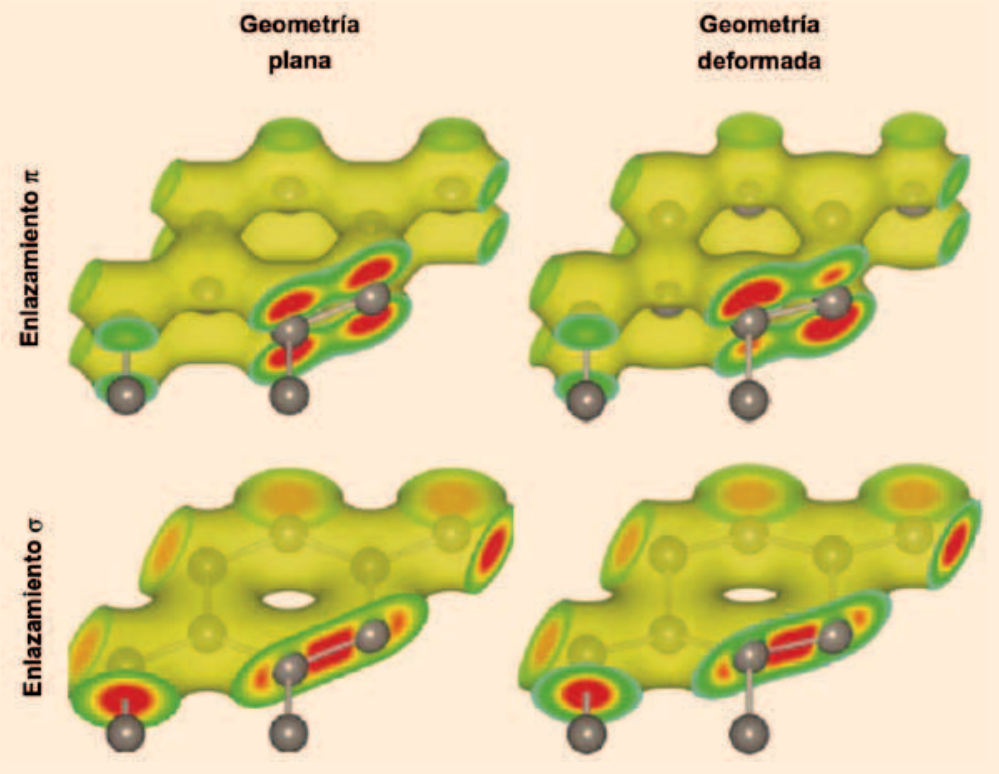

lo que genera un disminución en el grado de enlazamiento tipo $\sigma$ y $\pi$ como se observa en los mapas de densidad electrónica presentados en la figura 3 (Sahin et al., 2009).

Ahora, en cuanto a propiedades se refiere, este nuevo material bidimensional a base de silicio exhibe convenientemente varias características similares al grafeno. Esto abre un amplio abanico de nuevas posibilidades para este material, contrario a los pronósticos no tan esperanzadores que anteriormente se tenían reservados para este elemento.

El silicio cuenta con la ventaja por encima del carbono de ser un material conocido de forma muy extensa en la industria electrónica desde mediados del siglo pasado con la invención del transistor. En la actualidad, después de más de medio siglo, la gran mayoría de los procesos de producción de alta tecnología giran en torno a este elemento tan abundante y de fácil acceso.

Las propiedades electrónicas, magnéticas y químicas calculadas para el siliceno LB, muestran la misma tendencia que para el grafeno, lo cual hace del siliceno un candidato viable para dispositivos electrónicos. En lo particular, la estructura de bandas del siliceno, mostrada en la figura 4, exhibe un cruce lineal al nivel de Fermi entra la banda de valencia y la banda de conducción. Esto resulta en que los transportadores de carga del material exhiben un comportamiento típico de un fermion de Dirac, es decir, el comportamiento de una partícula sin masa. Además, la velocidad de Fermi calculada por Lew Yan Voon y colaboradores en el 2010 para el grafeno y el siliceno resulta en 6.3E5 y 5.1E5, respectivamente, velocidades bastante similares en magnitud (Lew Yan Voon et al., 2010). 
FIGURA 4. Estructura de bandas para el grafeno y siliceno. Imagen adaptada de Sahin et al., 2009.
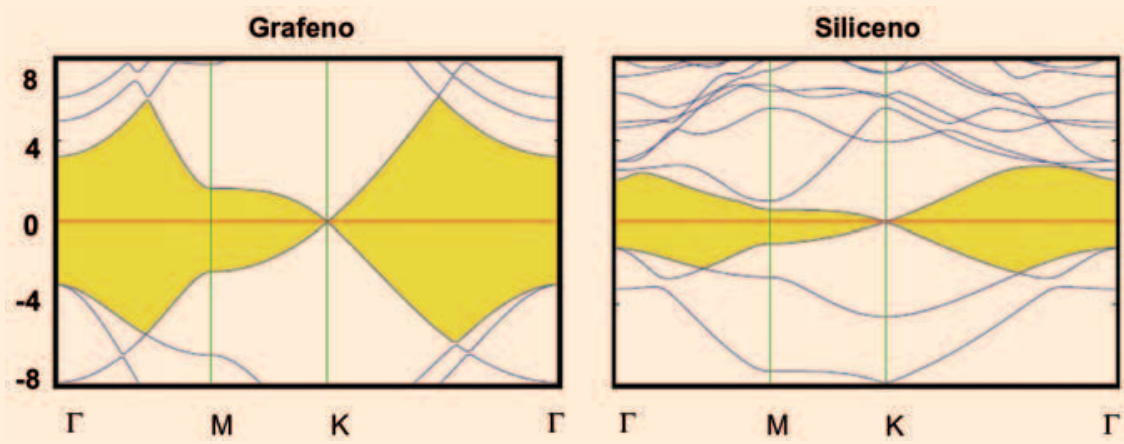

Debido a las asombrosas propiedades electrónicas que presenta el siliceno, se abre la posibilidad de fabricar en un futuro no muy lejano dispositivos electrónicos flexibles como: televisiones plegables, celulares que se enrollan y se llevan detrás de la oreja, dispositivos de lectura como en un libro tradicional y ropa con computadoras integradas, entre otros (Murray y Murray, 2012). Este tipo de dispositivos, en un futuro, conformarán una parte muy importante en la mercadotecnia de productos de alta tecnología que serán sumamente demandados por una sociedad que se encuentra inmersa en una constante evolución tecnológica.

Dada la gran cantidad de estudios teóricos que describen las propiedades tan interesantes del siliceno, se han realizado diversos experimentos con la pretensión de sintetizar su estructura mediante el uso de diferentes técnicas por medios físicos y químicos. Esto es debido a que el silicio prefiere, por sí solo, formar estructuras con configuración tetraedral en lugar de trigonal como sucede con el carbono, el cual puede adoptar ambas configuraciones con relativa facilidad.

Las primeras nanoestructuras bidimensionales de silicio que han sido sintetizadas fueron hechas por medio de métodos químicos basados en soluciones por Hideyuki Nakano y colaboradores del Instituto de Investigación del Grupo Toyota (Nakano et al., 2006). El experimento consistió en exfoliar el compuesto cristalino disiliciuro de calcio $\left(\mathrm{CaSi}_{2}\right)$, que está formado por un apilamiento alternado de planos atómicos de Ca y Si con estructura hexagonal. Sin embargo, como resultado del proceso, las nanoestructuras obtenidas (figura 5) se encontraban químicamente enlazadas con oxígeno, además de estar dopadas con magnesio como producto del procedimiento empleado.

Posteriormente, con el propósito de sintetizar hojas de siliceno libres de oxígeno y con tamaños laterales mayores, algunos investigadores trataron otros métodos de exfoliación partiendo de compuestos diferentes como lo es el polisilano (Okamoto et al., 2010).

Otros grupos de investigación, que últimamente han obtenido resultados muy interesantes y exitosos, abordaron el problema del crecimiento de siliceno mediante el empleo de depósitos de silicio por medio físicos con la selección de sustratos metálicos como es el caso de la plata. Dentro de los estudios experimentales más destacados 
FIGURA 5. Imagen obtenida por microscopía electrónica de transmisión en donde se observa una hoja de siliceno obtenida mediante la exfoliación del $\mathrm{CaSi}_{2}$. Imagen recuperada de Nakano et al., 2006.

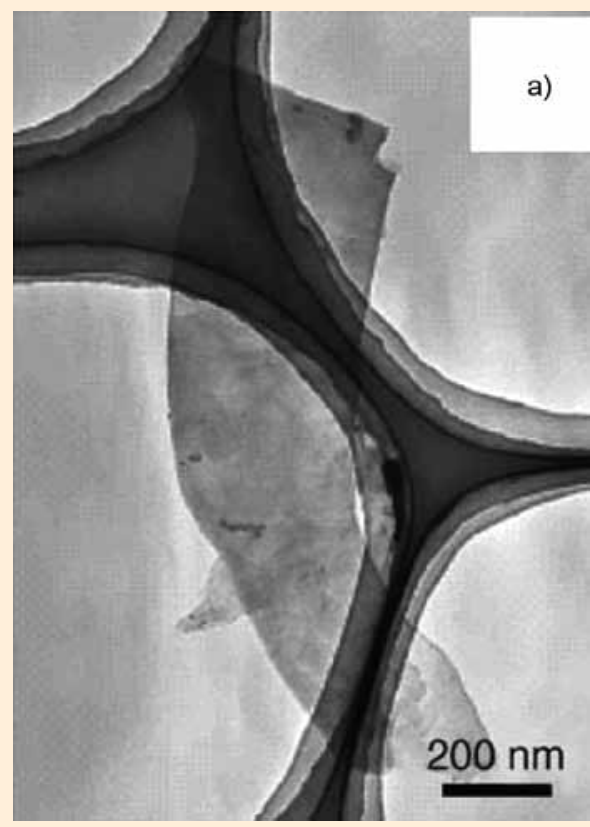

en relación con esta línea, se encuentra el trabajo presentado en 2012 por Chun-Liang Lin y colaboradores (Chun-Liang et al., 2012) en el que se muestran estudios teóricos y experimentales acerca del crecimiento de siliceno en una superficie de plata con orientación [111].

En tal experimento la fabricación de siliceno ha sido efectuada en una cámara de ultra alto vacío mediante el empleo de la técnica de depósito de vapores por medios físicos como lo es la sublimación de un blanco de silicio por calentamiento. En la figura 6 se aprecia el depósito de siliceno con la clara formación de una estructura hexagonal sobre una superficie de plata con orientación [111].

Además, mediante el empleo de sustratos de plata con orientaciones cristalográficas diferentes, por ejemplo la [110], ha sido posible crecer otro tipo de estructuras de siliceno en forma de listones.

Al momento, lo que han demostrado los resultados experimentales, contrario al grafeno que puede formar monocapas independientes, es que el siliceno sólo se ha logrado crecer exitosamente sobre sustratos metálicos de plata. Además, dada la tendencia del silicio a formar configuraciones tetraedrales, parece indispensable el uso de un sustrato para facilitar un crecimiento epitaxial en donde se copie la estructura cristalina de éste último.

En lo concerniente a la manipulación de las propiedades mostradas por el siliceno, existen diversos trabajos teóricos que han tratado de simular la incorporación de átomos de distintas especies, como hidrógeno, fluor y sodio en la superficie de siliceno. En los trabajos más destacados, se encuentran algunas investigaciones teóricas en 
FIGURA 6. Imagen obtenida por STM de la topografía de siliceno depositado sobre Ag [111]. Figura tomada de Chun-Liang Lin et al., 2012.

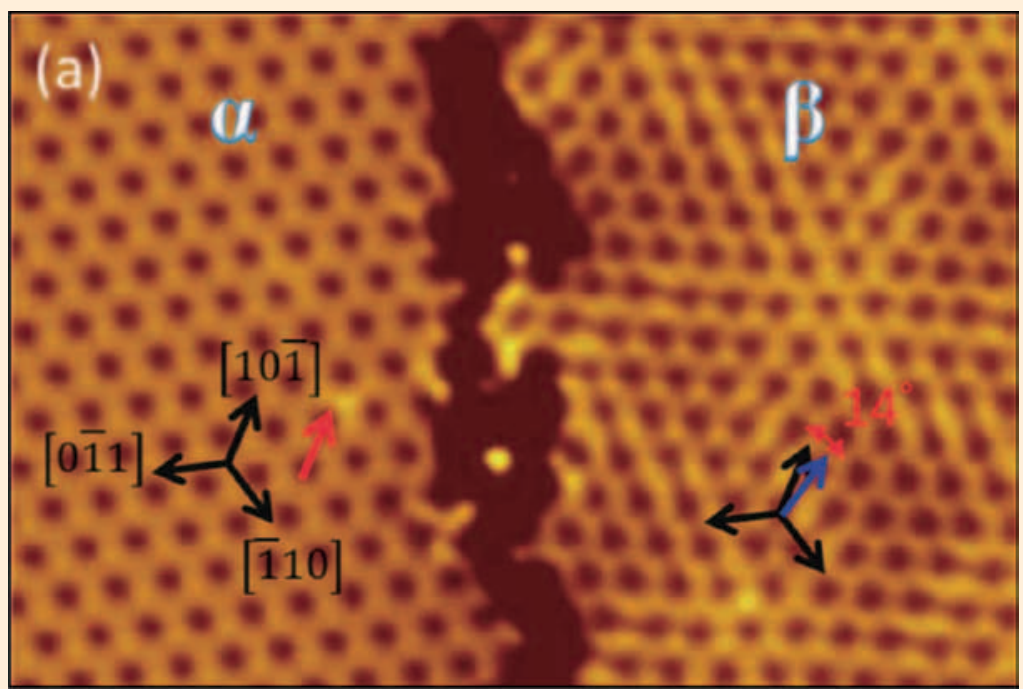

relación con la hidrogenación parcial y completa del siliceno que pasiva su superficie, como ha sido estudiado en 2011 por Osborn y colaboradores (Osborn et al., 2011). En dicha investigación, se encontró que, además de la pasivación obtenida, la estructura de bandas del siliceno puede ser modificada desde su estado inicial que muestra un comportamiento semimetálico hasta un comportamiento completamente aislante a través de distintos grados de hidrogenación.

Estudios todavía más recientes revelan que, dependiendo del grado de pasivación de la superficie y la disposición de los átomos de hidrógeno en ella, es posible inducir cierto grado de ferromagnetismo en el siliceno (Zhang et al., 2012).

Por otra parte, aunado a las novedosas posibilidades que ofrece el siliceno y los recientes descubrimientos en el campo de la electrónica molecular, existe la posibilidad no abordada aún de funcionalizar su superficie mediante la adición de moléculas orgánicas. Para abordar el tema de la funcionalización orgánica, nuestro grupo de investigación en el Centro de Nanociencias y Nanotecnología realiza simulaciones de la adsorción de hidrocarburos insaturados simples como son el acetileno, etileno y estireno sobre siliceno hidrogenado, empleando la teoría del funcional de la densidad (figura 7).

Mediante esta investigación de carácter teórico, se han estudiado las etapas de las reacciones correspondientes entre la molécula y la superficie, con el fin de determinar los perfiles de mínima energía y las barreras de activación para la adsorción de alquenos y alquinos sobre siliceno siguiendo el mecanismo de una reacción en cadena. Los resultados obtenidos se han comparado con los ofrecidos por la superficie hidrogenada del silicio cristalino en una orientación cristalográfica [111]. El Si [111], además de que presenta una configuración bastante similar en superficie a la del siliceno, ha sido estudiado teórica y experimentalmente desde la década de los noventa para propósitos de la funcionalización orgánica. 
Mundo Nano | Artículos | Vol. 6, No. 10, enero-junio, 2013 | www.mundonano.unam.mx

FIGURA 7. Ilustración esquemática de moléculas de acetileno, etileno y estireno adsorbidas en siliceno hidrogenado. Los colores mostaza, azul y blanco representan los átomos de $\mathrm{Si}$, $\mathrm{C} \mathrm{e} \mathrm{H}$, respectivamente.
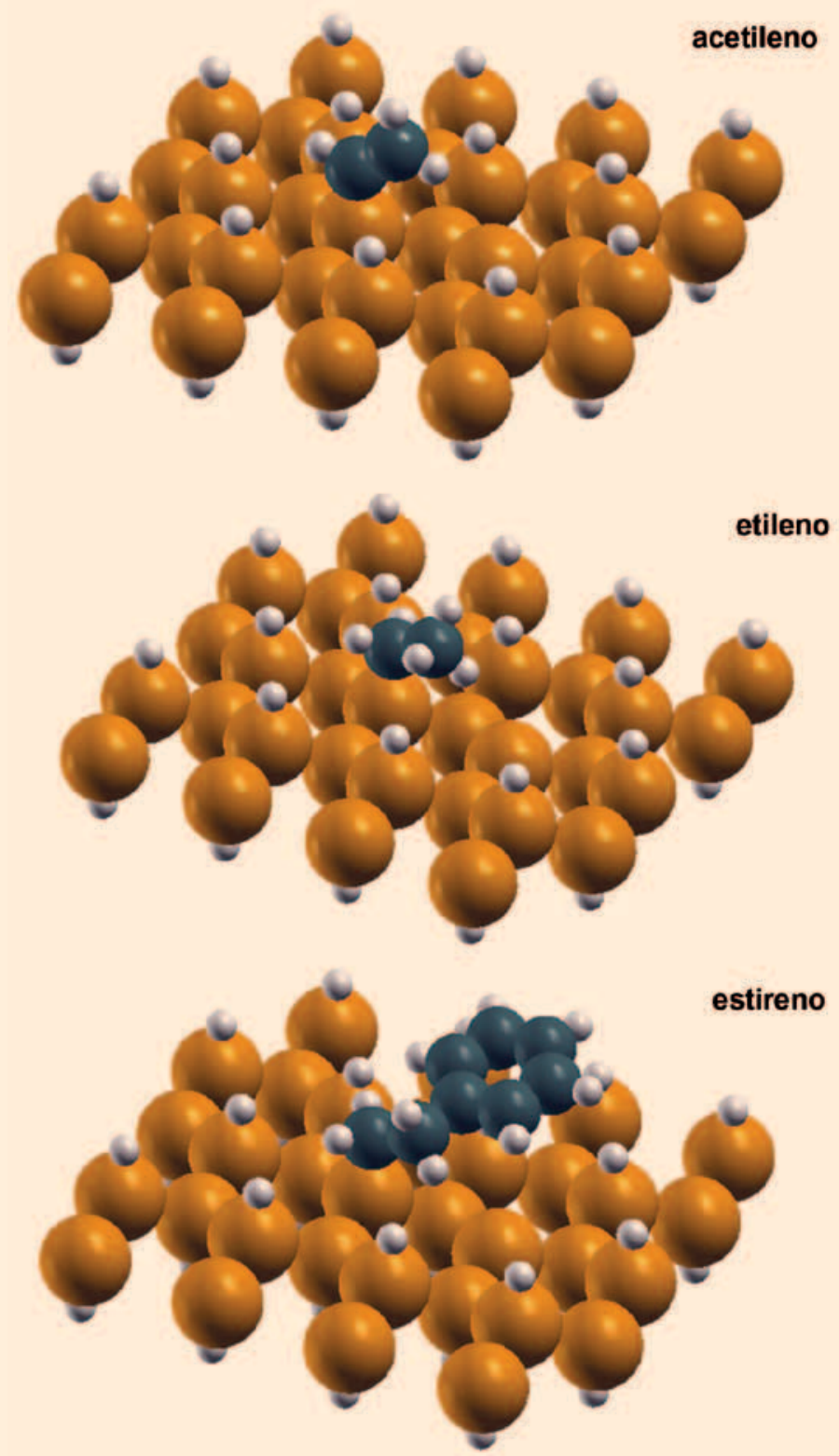

estireno 
Los resultados obtenidos al momento muestran que es posible el desarrollo de la reacción de adsorción de estireno sobre siliceno con la misma viabilidad que sobre la superficie de Si [111], esta última estudiada bajo las mismas condiciones en 2004 por nosotros (Takeuchi et al., 2004). En el caso de la adsorción de moléculas más pequeñas, como es el caso de acetileno y etileno, se encontró que las etapas de la reacción de adsorción y las barreras de activación son energéticamente más favorables para el siliceno que para el Si [111]. Estos resultados posicionan al siliceno como un sistema que presenta una mayor viabilidad para la adsorción de moléculas insaturadas de hidrocarburos simples en comparación con el silicio cristalino.

En resumen, el siliceno, un material que recientemente ha aparecido en la red científica como una novedosa faceta del silicio, representa un gran nicho de oportunidades de investigación tanto teóricas como experimentales, debido a que presenta propiedades análogas a las del grafeno, además de que dichas propiedades se pueden manipular mediante la incorporación de diferentes elementos. Con la aparición de este novedoso material bidimensional y con el desarrollo de métodos que permitan su fabricación de una forma mucho más eficiente, es posible que la tecnología del silicio permanezca en la industria electrónica por mucho más tiempo del que anteriormente se creía posible, mediante la fabricación de una siguiente generación de novedosos dispositivos electrónicos flexibles a base de siliceno.

\section{REFERENCIAS BIBLIOGRÁFICAS}

Cahangirov, S.; Topsakal, M.; Aktürk, E.; Sahin H. y Ciraci, S. (2009) “Two and one dimensional honeycomb structures of silicon and germanium". Physical Review Letters. 102. 236804.

Chun-Liang, Lin; Ryuichi, Arafune; Kazuaki, Kawahara; Noriyuki, Tsukahara; Emi, Minamitani; Yousoo, Kim; Noriaki, Takagi y Maki, Kawai. (2012) "Structure of silicene grown on Ag (111)". Applied Physics Express. 5. 045802.

Lew Yan Voon, L. C.; Sandberg, E.; Aga, R. S. y Farajian, A. A. (2010) “Hydrogen compounds of group-IV nanosheets". Applied Physical Letters. 97. 163114.

Martínez Guerra, E.; Cifuentes Quintanal, M.E.; de Coss, R. (2009) “Grafeno: un paso hacia el futuro". Mundo Nano. 1: 15-23.

Murray Tortarolo, G. y Murray Prisant, G. (2012) “Grafeno ¿La siguiente revolución tecnológica?”. ¿cómo ves? Revista de divulgación de la ciencia de la Universidad Nacional Autónoma de México. 164: 22-25.

Nakano, H.; Mitsuoka, T.; Harada, M.; Horibuchi, K.; Nozaki, H.; Takahashi, N.; Nonaka, T.; Seno Y. y Nakamura H. (2006) "Soft synthesis of single-crystal silicon monolayer sheets". Angewandte Chemie-International Edition. 45: 6303-6306.

Novoselov, K. S.; Geim, A. K.; Morozov, S. V.; Jiang, D.; Katsnelson, M. I.; Grigorieva, I. V.; Dubonos, S. V.; Firsov, A. A.; (2005) “Two-dimensional gas of massless Dirac fermions in grapheme". Nature. 438: 197-200.

Osborn, T. H.; Farajian, A. A., Pupysheva, O. V.; Aga, R. S. y Lew Yan Voon, L. C. (2011) “Ab initio simulations of silicene hydrogenation". Chemical Physics Letters. 511: 101-105.

Sahin, H.: Cahangirov, S.; Topsakal, M.; Bekaroglu, E.; Akturk, E.; Senger, R. T. y Ciracli, S. (2009) "Monolayer honeycomb structures of group-IV elements and III-V binary compounds: First-principles calculations". Physical Review B. 80: 155453. 
West, R.; Mark, J. y Michil, J. (1981) “Tetramesityldisilene, a stable compound containing a silicon-silicon double bond". Science. 214: 1343-1344.

Takeda, K. y Shiraishi, K. (1994) "Theoretical possibility of stage corrugation in Si and Ge analogs of graphite”. Physical Review B. 50: 14916-14922.

Takeuchi, N.; Kanai, Y. y Selloni, A. (2004) "Surface reactions of alkynes and alkenes with H-Si(111): a density functional study". Journal of the American Chemical Society. 126: 15890-15896.

Zhang, P.; Li, X. D.; Hu, C. H.; Wu, S. Q., y Zhu, Z. Z. (2012) “First-principles studies of the hydrogenation effects in silicene sheets". Physics Letters A. 376: 1230-1233. 